Bangladesh Journal of Anatomy January 2010, Vol. 8 No. 1 pp. 34-38

\title{
Effect of Gestational Diabetes Mellitus on Gross Morphological Structure of Preterm Placenta
}

\author{
Fahima Akhter ${ }^{1}$, Mst. Laila Anjuman Banu², Roxana Ferdausi ${ }^{3}$
}

\begin{abstract}
:
Context: Preterm birth is the major cause of perinatal mortality and morbidity. For the last few decades, it has become an important issue in public health policies of developing countries. Gestational diabetes mellitus (GDM) is one of the high-risk factors for the preterm birth and altered fetal development. This pregnancy induced disorder leads to an increased level of all metabolic substances to the fetal circulation due to development of maternal insulin resistance. It imposes a heavy burden on the mother who is pregnant and these patients have a tendency toward metabolic instability. As there is an intimate relationship between the fetus and placenta, the present study aimed to observe the effect of this pregnancy induced disorder to the preterm placenta.
\end{abstract}

Study design: The study was observational, analytical and cross sectional.

Place and period of study: The study was carried out in the Department of Anatomy, BSMMU, Dhaka during the study period of January 2005 to December 2005.

Materials and Methods: A total of forty-four samples were collected from women during 28 weeks to 36 completed weeks of gestation. Among them, twenty-two samples belonged to mothers having GDM and twenty-two belonged to normal pregnancy (control group). The placentas were examined to measure their diameter, thickness, cotyledons number, weight, and volume.

Result: In this study, the GDM group showed significantly higher values for the variables of diameter, weight and volume. On the other hand, the thickness of the placenta showed lower values and cotyledons number showed higher values in GDM group but the result did not reach a significant level.

Conclusion: The findings in this study supported that the gross morphological structure of the placentas in GDM mother did not present any specific, constant or uniform pattern of abnormality. Therefore, it is difficult to establish a clear cut correlation between the placental changes and diabetic state in the mother during pregnancy. However, increased placental weight, volume and diameter found in gestational diabetic mother have supported that these changes may be a long term compensatory mechanism, aiming to secure a sufficient nutrient supply to support the growth of large-for-gestational age (LGA) fetus. But the hormonal and metabolic abnormalities present in the diabetic mother and the fetus are important variables to be considered when studying the placenta.

Key -words: Preterm Placenta, GDM, Gross morphology.

Introduction

Preterm birth accounts for $6 \%$ to $10 \%$ of all birth and it is a major cause of perinatal morbidity and mortality ${ }^{1}$.Among the different causes, gestational diabetes mellitus (GDM) is an important factor for

1. Assistant Professor, Department of Anatomy, Enam Medical College, Savar, Dhaka.

2. Associate Professor of Anatomy, BSMMU, Dhaka

3. Professor \& Head, Department of Anatomy, Anwer Khan Modern Medical College, Dhaka.

Correspondence: Dr. Fahima Akhter preterm birth where both mother and fetus are in a vulnerable state. Approximately $2 \%$ to $3 \%$ of pregnancies are affected by diabetes mellitus, 90\% of which represents gestational diabetes mellitus ${ }^{2}$. Diabetes and pregnancy is associated to the fetus in terms of malformations and altered fetal growth, that is related to the increased perinatal morbidity and mortality ${ }^{3}$.

The placenta is an organ that is absolutely essential to the survival of the fetus. The term 'placenta' was used for the first time in 1559 and all the enabolites 
needed for fetal metabolism come from the mother's blood and fetal catabolites are passed back into the mother's circulation through the placenta ${ }^{4}$. So, as a mirror, it reflects the intra-uterine status of the fetus. Its metabolic functions are complex, and it undergoes continuously throughout gestation a change in weight, structure, shape and function in order to support prenatal life ${ }^{5}$. In GDM, when the intra-uterine environment for fetus become hostile, the placenta tries to exert its reserve capacity by changing its morphological structure, as well as some pathological changes occur that are compounded principally of some disturbances in its normal rate of maturation ${ }^{6}$. So, gross morphological study of the placenta in GDM may provide information of certain alterations which could reflect the fetal condition and which would be of value in terms of predicting fetal outcome.

\section{Materials and methods}

Forty-four placentas were selected from Bangladeshi women who gave birth to a single alive baby through caesarean section during 28 weeks to 36 completed weeks of gestation. Specimens were collected from Bangladesh Institute of Research in Diabetic, Endocrine and Metabolic Disorders (BIRDEM) Dhaka, and Bangabondhu Sheikh Mujib Medical University (BSMMU) Dhaka, from June 2005 to October 2005. All the placentas were collected as soon as possible after delivery from the obstetric operation theatre. The study was done in the department of Anatomy, BSMMU, Dhaka, during the study period of January 2005 to December 2005.

Among Forty-four placentas, twenty-two were collected from non-diabetic mothers and were considered as Control group, and the remaining twenty- two had GDM . Mothers with long standing diabetes mellitus or hypertension, or preeclampsia, Rh-negative blood group, Positive ANA (Anti-nuclear antigen), Positive VDRL, multiple gestations in current pregnancy, severely anemic $(\mathrm{Hb} \%<7 \mathrm{gm} /$ dl) as well as mothers delivering congenitally malformed babies were excluded from the study.

The placentas were collected in labeled plastic bags. Immediately after collection, the following gross morphological variables of each placenta for two groups were studied:-
1. Diameter of the placenta - The placentas were kept on a flat tray and membranes were trimmed from their edges. Blood was removed gently from both surfaces with cotton wool and the umbilical cord was cut about $2 \mathrm{~cm}$ proximal of its insertion ${ }^{7}$. Then the maximum diameter of each placenta was measured with a metallic scale graduated in centimeters $(\mathrm{cm})$. At right angles to the first one, the second maximum diameter was recorded (shown in figure -1). Then the mean of these two measurements was considered as the diameter of each placenta ${ }^{8}$.

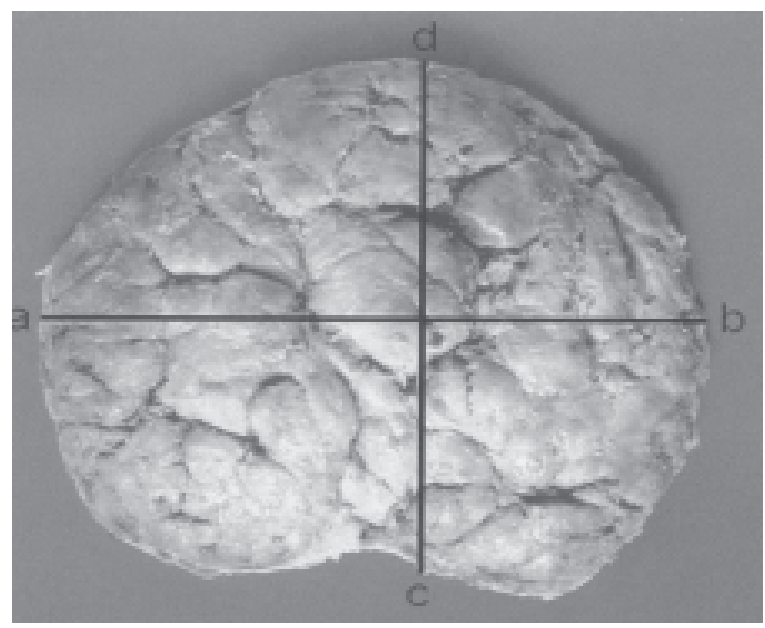

Fig.-1: Photograph of the maternal surface of a placenta showing the procedure of measurement of its diameters. Line ab shows the apparently maximum diameter, while line cd shows the apparently maximum diameter at right angles to line $a b$.

2. Thickness of the placenta - Placental thickness was measured by piercing a large needle through five points. To do this, each placenta was arbitrarily divided into three equal zones; central, middle and peripheral by drawing two imaginary circles on its maternal surface, keeping the center of placenta as an axis ${ }^{8}$ (shown in figure -2).

3. Number of cotyledons - Placenta was taken on both hands facing the fetal surface upward. Then gentle pressure was applied from the central part of the fetal surface to periphery with the thumbs of both hands while the peripheral part was holded by the other fingers. This 


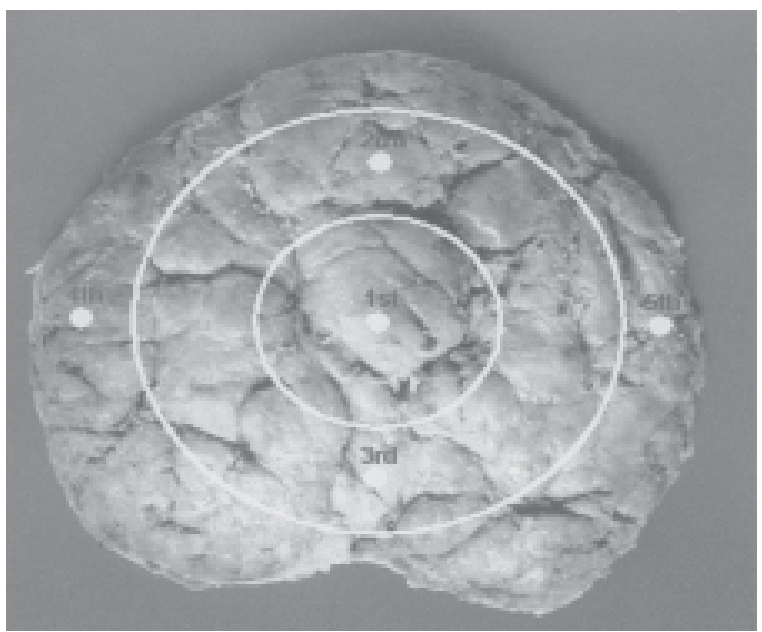

Fig.-2: Photograph of the maternal surface of a placenta with division into three zones showing five points for the measurement of its thickness.

maneuver caused separation of the cotyledons to make them prominent in the maternal surface. Then it was put on a tray with maternal surface facing upwards by placing a woodenblock on the fetal surface. Counting was started from left side of one end and going through right ward. In this way, counting was continued in spiral manner ${ }^{8}$ (shown in figure -3).

4. Weight of the placenta - After recording the above variables, each placental weight was recorded with a weighing machine in grams $(\mathrm{g})$.

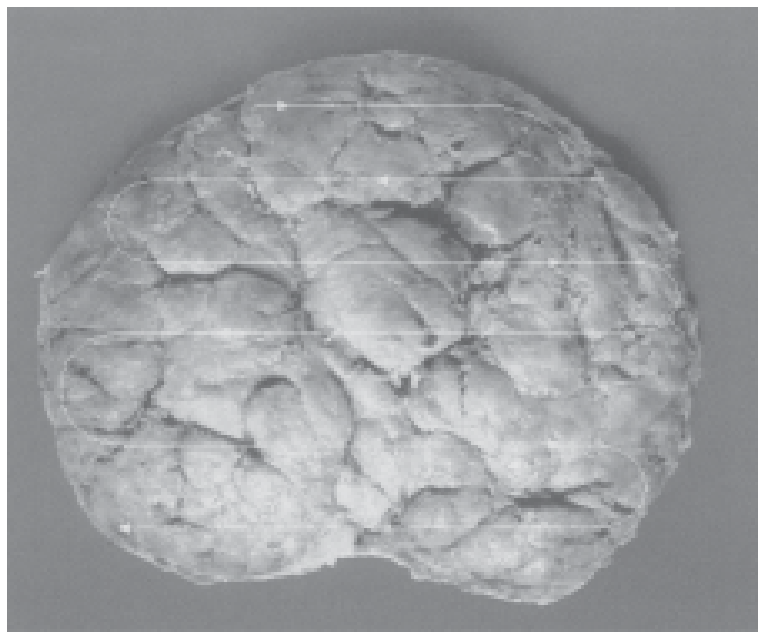

Fig.-3: Photograph of the maternal surface of a placenta showing the procedure of counting the number of cotyledons.
5. Volume of the placenta - The volume of the placenta was measured by immersing it in water in a plastic bucket to which a plastic drawingtube was attached. The displaced water drained through the tube was collected in a container and measured in a graduated cylinder in milliliters $^{9}(\mathrm{ml})$.

Results were obtained by calculating means, standard deviations (SD) and analyzing significant difference using a computer- based program (SPSS, version II). The post Hoc option of analysis of variance (ANOVA) was done to compare two groups for every variable. The difference was considered to be significant statistically at $5 \%$ level (i.e. $P<0.05$ ).

\section{Results}

In this study, Table- I showed that the placental weight, volume and diameter had significantly higher values in GDM group when compared to the control group. Thickness of the placenta and their number of cotyledons did not differ significantly in between two groups. However, mean values of the placental thickness was less and mean values of number of cotyledons was more in GDM group than control group. Figure- 4 shows multiple bar diagram showing frequency distribution of placental weight $(\mathrm{gm})$ in gestational diabetes mellitus and control groups. Here $10 \%$ placentas of control group are within 100$200 \mathrm{gm}$ where in GDM group no placentas were found within this range. Again, maximum 55\% placentas in GDM group are above $400 \mathrm{gm}$, whereas maximum $45 \%$ placentas in control group are above these values. This indicates that incidence of higher weighted placentas is more in the GDM group than that of control group.

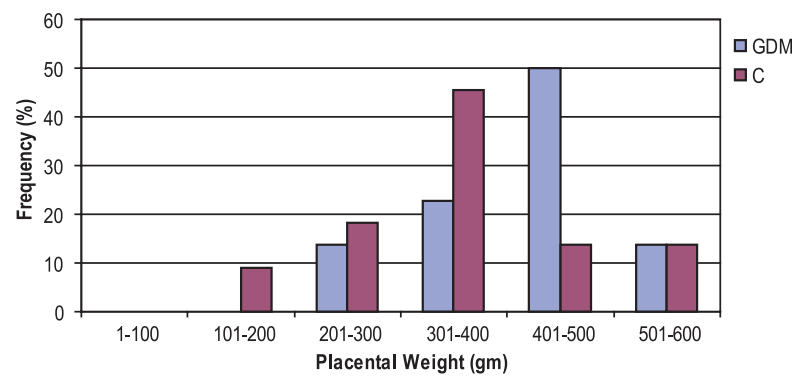

Fig.-4: Multiple bar diagram showing frequency distribution of placental weight (gm) in gestational diabetes mellitus and control groups. 
Bangladesh J. Anat. 2010; 8(1) : 34-38

Table -I

Macroscopic variables of the preterm placenta in two groups

\begin{tabular}{|c|c|c|c|}
\hline Variable & $\begin{array}{l}\text { Control (C) } \\
\qquad(n=22)\end{array}$ & $\begin{array}{l}\text { GDM (G) } \\
(n=22)\end{array}$ & $\begin{array}{l}{ }^{*} \text { Statistically significant } \\
\text { two groups (when } \mathrm{P}<0.05 \text { ) }\end{array}$ \\
\hline \multicolumn{4}{|l|}{ Weight (gm) } \\
\hline Mean \pm SD & $(361.68112 .62)$ & (41797.32) & \\
\hline Range & $150-580$ & $151-580$ & C vs G: ** \\
\hline \multicolumn{4}{|l|}{ Volume (ml) } \\
\hline Mean SD & (341.82103.95) & $(415.0099 .37)$ & \\
\hline Range & $125-500$ & $140-650$ & C vs G: ** \\
\hline \multicolumn{4}{|l|}{ Thickness (cm) } \\
\hline Mean SD & (1.360.53) & $(1.330 .45)$ & \\
\hline Range & $0.57-2.37$ & $0.70-2.20$ & \\
\hline \multicolumn{4}{|l|}{ Diameter (cm) } \\
\hline Mean SD & $(17.412 .97)$ & (19.202.30) & \\
\hline Range & $7.75-22.50$ & $12.5-22.50$ & C vs G: ** \\
\hline \multicolumn{4}{|l|}{ No. of cotyledons } \\
\hline Mean SD & (17.953.43) & (18.555.07) & \\
\hline Range & $12-24$ & $8-26$ & \\
\hline
\end{tabular}

The Post Hoc option of analysis of variance (ANOVA) was done to compare each group with other group for each variable. The differences between two groups that were statistically significant (i.e., $\mathrm{P}<0.05$ ) are presented here as **.

\section{Discussion}

A wide variety of morphological changes have been reported on placentas of diabetic women. In general, placenta from diabetic women tends to be heavier than the general population at the same gestational age $^{10-14}$. Teasdale ${ }^{3}$ stated that a significant accumulation of non-parenchymal tissue and only a moderate increase in parenchymal tissue may be the cause of heavier placenta in GDM. On the other hand, it was due to a parallel increase in parenchymal and non-parenchymal tissue and in respect of parenchymal components, there was increase in the surface area of exchange between mother and fetus, in terms of peripheral villi and capillary surface areas and intervillous space volume ${ }^{15}$. However, regarding the cause of increased placental weight in diabetic mother, it was suggested that it was due to increased proliferation of the placental tissues reflected by a higher DNA contents $^{16}$. So, increased placental growth is a consequence of a co-existing metabolic or endocrine effect of hyperinsulinaemia and it would be logical to assume that the less severe and better controlled diabetic state of the patient, the less striking would be the placental abnormalities and it was reflected on the observation of Clarson et $\mathrm{al}^{17}$ who found no increase in mean placental weight in women with well controlled diabetes mellitus.

The total volume of the placentas of diabetics was on average $12 \%$ larger than that of the non diabetics stated by Boyd et $\mathrm{al}^{18}$. In the present study, the increased volume of the placenta was also observed in the GDM group and this increased placental volume may be the consequences of increased volume of the parenchymal components of the 
placental tissues ${ }^{11}$. Mayhew et al. ${ }^{19}$ also observed that the volume of fetal capillaries was $45 \%$ greater in diabetes and the expansion of the fetal blood space in the peripheral villi of diabetic placenta was associated with a $39 \%$ expansion of their surface area, that ultimately may be responsible for the increased total volume of the placenta. On the other hand, significantly increased diameter of placenta in diabetic mother may correlate with their increased volume and increased number of cotyledons as compared to the Control group. However, further investigations can be recommended to search for placental structural changes in gestational diabetic mothers that are significantly related to the preterm birth.

\section{References}

1. Germain HE. Preterm labor: Placental pathology and clinical correlation 1999; 2849.

2. Haver MC, Gregory J, Locksmith, Emmet E. Irregular menses: An independent risk factor for gestational diabetes mellitus. Am J Obstet Gynecol 2003; 188: 1189-91.

3. Teasdale F. Histomorphometry of the human placenta in class $B$ diabetes mellitus. Placenta 1983; 4: 1-12.
4. Udainia A, Bhagwat SS, Mehta CD. Relation relevance. J Anat Soc" India 2004; 53(1): 27 30.

5. Teasdale F. Gestational changes in the functional structure of the human placenta in class relation to fetal growth: a morphometric study: Am J Obstet Gynecol 1980; 137: 5608.

6. Fox H. Pathology of the placenta in maternal diabetes mellitus. Obstet Gynecol 1969; 34: 792-8.

7. Laga EM, Driscoll SG, Manro HN. Quantitative studies of human placenta: morphometry. Biol Neonate 1973; 23: 231-59.

8. Anwar S. Histomorphological changes of human placenta in diabetes mellitus [Thesis]. Dhaka: Department of Anatomy, University of Dhaka; 1999.

9. Clavero JA, Botella Llusia J. Measurement of the villus surface in normal and pathologic placentas. Am J Obstet Gynecol 1963; 86: 234-40.

10. Driscoll SG. The pathology of pregnancy complicated by diabetes mellitus. Med Clin North Am 1965; 49: 1053-67.

11. Teasdale F. Histomorphometry of the placenta of the diabetic woman: class A diabetes mellitus. Placenta 1981; 2: 241-52. 